\title{
Trauma renal: ensaio pictórico dos principais achados tomográficos
}

\section{Renal trauma: pictorial essay of the main computed tomography findings}

Francisco José Rodrigues de Moura Filho ${ }^{1}$. Marilza Oliveira ${ }^{1,2}$. Ana Maria Araújo Martins ${ }^{2}$. Pedro Henrique Sales Pontes². Mário Márcio Fernandes Barros².

1 Médico(a) Radiologista, Instituto Dr José Frota (IJF), Fortaleza, Ceará, Brasil. 2 Médico(a) Radiologista, Instituto São Carlos de Ensino e Pesquisa, Fortaleza, Ceará, Brasil.

\section{RESUMO}

O rim é o terceiro órgão mais acometido em pacientes vítimas de trauma abdominal. A maioria das lesões traumáticas renais são pequenas, sendo tratadas conservadoramente. A indicação absoluta de tratamento cirúrgico é reservada à menor parte dos casos $(<5 \%)$. A tomografia computadorizada é a ferramenta diagnóstica mais importante para adequada avaliação da lesão renal e classificação segundo os critérios da Associação Americana para a Cirurgia do Trauma (AAST). No presente estudo, nós descrevemos os achados tomográficos de cinco pacientes vítimas de trauma renal atendidos em um hospital terciário de referência em trauma e emergência do estado do Ceará e classificamos as lesões renais encontradas segundo os critérios da AAST.

Palavras-chave: Trauma. Tomografia computadorizada. Rins.

\section{ABSTRACT}

The kidney is the third most affected organ in patients victim of abdominal trauma. The majority of traumatic renal lesions are minor injuries being treated conservatively. The absolute indication of surgical treatment is reserved for a smaller number of cases $(<5 \%)$. Computed tomography (CT) is the most important diagnostic tool for adequate evaluation of renal injury and classification according to the criteria of the American Association for Trauma Surgery (AAST). In the present study, we described the computed tomography findings of five renal trauma patients treated at a tertiary hospital that is reference in trauma and emergency in the state of Ceará, and we also classified the renal lesions found according to the AAST criteria.

Keywords: Trauma. Computed tomography. Kidneys.

Autor correspondente: Francisco José Rodrigues de Moura Filho, Rua Barão do Rio Branco 1816, Centro, Fortaleza, Ceará. CEP: $60025-061$. Telefone: +55 85 3255-5036. E-mail: drmouramd@yahoo.com

Conflito de interesses: Não há qualquer conflito de interesses por parte de qualquer um dos autores.

Recebido em: 15 Jun 2017; Revisado em: 24 Set 2017; Aceito em: 24 Set 2017. 


\section{INTRODUÇÃO}

Os rins são acometidos em 8 a 10\% dos pacientes que sofrem lesões abdominais no trauma fechado. Hematúria é um importante sinal clínico de lesão do trato urinário. As lesões que não necessitam de tratamento cirúrgico são as mais comuns e correspondem às contusões, hematomas subcapsulares, pequenas lacerações com hematomas perinefréticos limitados e pequenos infartos corticais. Já as lesões que requerem tratamento cirúrgico incluem fragmentação renal e lesão do pedículo vascular renal. ${ }^{1,2}$
A tomografia computadorizada é o método diagnóstico padrão ouro na avaliação do trauma renal, ${ }^{3,4}$ devendo ser realizada rotineiramente com o uso de contraste endovenoso, quando não há contraindicações, e permite classificar as lesões renais em cinco graus de acordo com a Organ Injury Scaling (OIS) da Associação Americana para a Cirurgia do Trauma (AAST) publicada em 1989, com a ultima atualização em 2016 (Tabela 1).

Tabela 1. Classificação do Trauma Renal - AAST.

\begin{tabular}{|c|c|c|}
\hline Grau & Tipo & Descrição \\
\hline I & Contusão & Hematúria micro ou macroscópica, estudos urológicos normais. \\
\hline I & Hematoma & Subcapular, não expansível, sem laceração do parênquima. \\
\hline II & Hematoma & Não expansível, perirrenal, confinado ao retroperitônio. \\
\hline II & Laceração & $<1 \mathrm{~cm}$ de profundidade do parênquima, sem extravasamento urinário. \\
\hline III & Laceração & >1 cm de profundidade do parênquima, sem ruptura do sistema coletor ou extravasamento urinário. \\
\hline IV & Vascular & $\begin{array}{l}\text { Lesão da artéria e veia renal principal com hemorragia controlada. Infartos segmentares sem } \\
\text { lacerações associadas. }\end{array}$ \\
\hline IV & Laceração & Laceração do parênquima que se estende até o sistema coletor. \\
\hline IV & Hematoma & Subcapsular expansível comprimindo o rim. \\
\hline V & Laceração & Rim fragmentado (shattered kidney). Avulsão ureteropélvica (JUP). \\
\hline $\mathrm{V}$ & Vascular & Avulsão ou trombose vascular com desvascularização do rim. \\
\hline
\end{tabular}

No estudo tomográfico, o hematoma subcapsular agudo pode ser caracterizado por uma imagem lentiforme, com material de alta atenuação (40-90UH), comprimindo o parênquima renal subjacente. ${ }^{4}$ A contusão renal pode ser observada como uma área focal do parênquima de baixa atenuação, sem realce na fase contrastada, associada a aumento localizado ou difuso do rim. Já a laceração aparece como faixas irregulares laminares de baixa atenuação dentro do parênquima renal, havendo extravasamento de urina na presença de comprometimento do sistema coletor. ${ }^{1,5}$ Infartos aparecem como áreas em cunha no parênquima renal, que se estende da medula à cortical com base periférica, de baixa atenuação e sem realce. Em alguns casos pode ser identificado contrastamento cortical, por irrigação colateral (nefrograma cortical $=$ sinal do anel cortical) ${ }^{5,6}$

A falência do rim em se opacificar (ausência de efeito nefrográfico) após a administração do contraste endovenoso é uma evidencia de lesão do seu suprimento vascular, seja decorrente de trombose ou ruptura. Assim, trombos ou coágulos na artéria renal cursam com nefrograma ausente, ao passo que trombos ou coágulos na veia renal cursam com um nefrograma persistente. Já as imagens tardias do estudo tomográfico permitem o diagnóstico de lesões do sistema coletor através do extravasamento de urina contrastada no seio renal e no espaço perirrenal medial. ${ }^{1,7,8}$

Considerando que o Instituto Dr. José Frota (IJF) é o maior hospital de urgência e emergência e referência no atendimento de pacientes vitimas de trauma do estado do Ceará e conta com um Centro de Imagem equipado com um Tomógrafo Multislice com 16 canais, que funciona 24 horas por dia, é de inquestionável valor para a comunidade científica a divulgação dos dados referentes ao diagnóstico por imagem deste grupo de pacientes, tendo assim o presente trabalho o objetivo de relatar os achados tomográficos de cinco casos de trauma renal atendidos no Instituto Dr. José Frota (IJF).

\section{DESCRIÇÃO DOS CASOS}

Os autores analisaram as imagens, de modo retrospectivo, no Centro de Imagem do Instituto Dr. José Frota (IJF), após aprovação no Comitê de Ética e Pesquisa deste hospital, de cinco casos de pacientes vítimas de trauma renal atendidos no IJF que foram submetidos ao estudo tomográfico contrastado, cujo protocolo empregado no serviço envolvia cortes tomográficos com $5,0 \mathrm{~mm}$ de espessura, incremento de 5,0 mm, com reconstruções multiplanares e volumétricas tridimensionais tendo sido adquiridas para o estudo urodinâmico fases pré-contraste, arterial, venosa e tardia. A apresentação de cada caso tem o objetivo de descrever os achados tomográficos encontrados e classificar as lesões renais de acordo com a Organ Injury Scaling (OIS) da Associação Americana para a Cirurgia do Trauma (AAST). 


\section{Caso 1}

Paciente de 19 anos, sexo masculino, vítima de lesão por arma branca em região lombar (Figura 1).

Achados Tomográficos: Laceração do polo inferior do rim esquerdo acometendo o parênquima (cortical e medular) com mais de $1 \mathrm{~cm}$ de profundidade. Hematoma subcapsular em crescente (não expansível) no rim esquerdo. Sem sinais de extravasamento de urina.

Classificação: Grau III.

Figura 1. Lesão renal grau III.

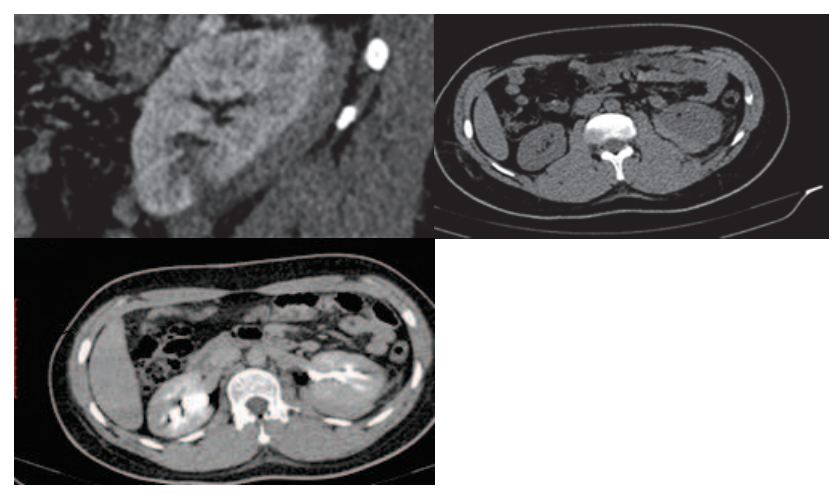

\section{Caso 2}

Paciente de 39 anos, sexo masculino, vítima de lesão por vários projéteis de arma de fogo (Figura 2).

Achados Tomográficos: Laceração bilateral - do polo inferior do rim esquerdo e terço médio do rim direito. Hematoma não expansível no rim direito e expansível no rim esquerdo. Sem extravasamento de urina.

Classificação: Grau IV.

Figura 2. Lesão renal grau IV.

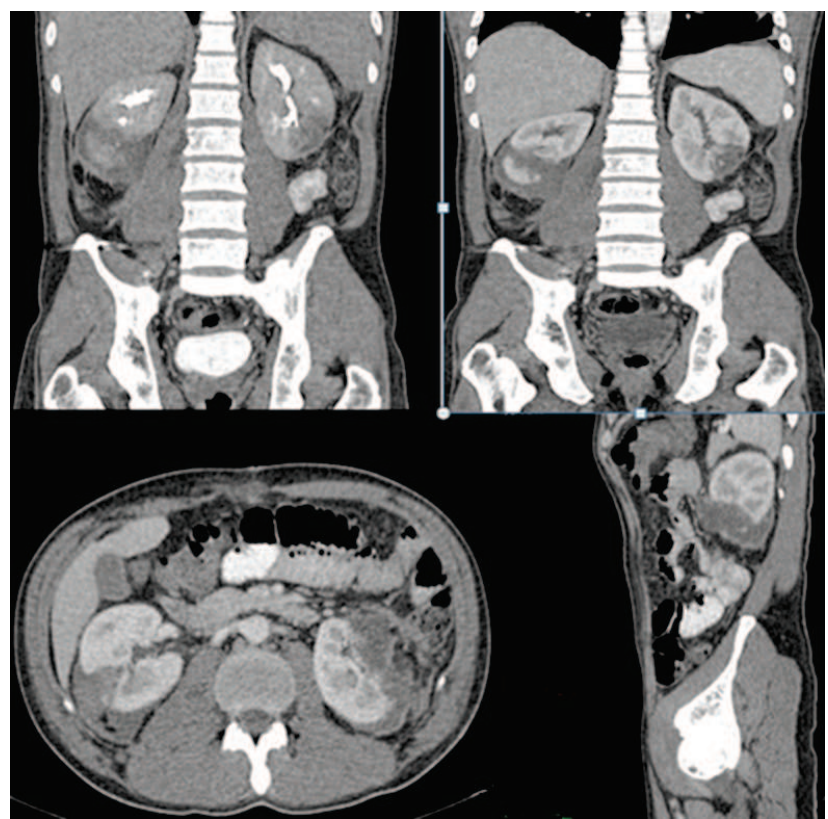

\section{Caso 3}

Paciente de 67 anos, sexo masculino, vitima de acidente motociclístico (Figura 3).

Achados Tomográficos: Laceração do polo inferior do rim direito acometendo todo o parênquima (cortical e medular). Hematoma contido pelos planos fasciais. Sangramento em diferentes tempos de evolução.

Classificação: Grau IV.

Figura 3. Lesão renal grau IV.

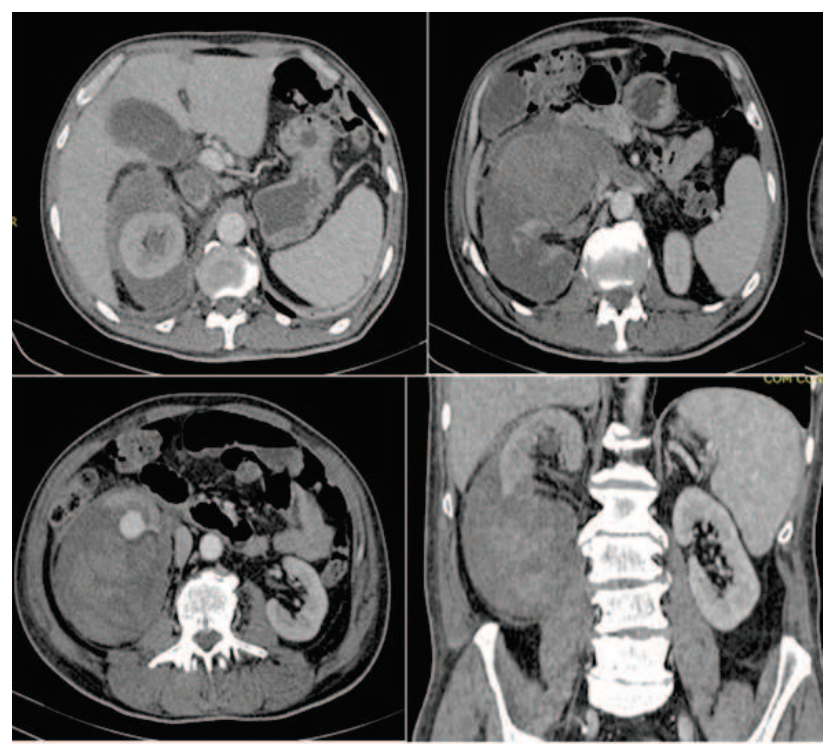

\section{Caso 4}

Paciente de 25 anos, sexo masculino, vítima de acidente motociclístico (Figura 4).

Achados Tomográficos: Infarto no rim esquerdo, polo superior. Lesão em cálice superior com extravasamento de urina, identificado na fase tardia.

Classificação: Grau IV.

Figura 4. Lesão renal grau IV.

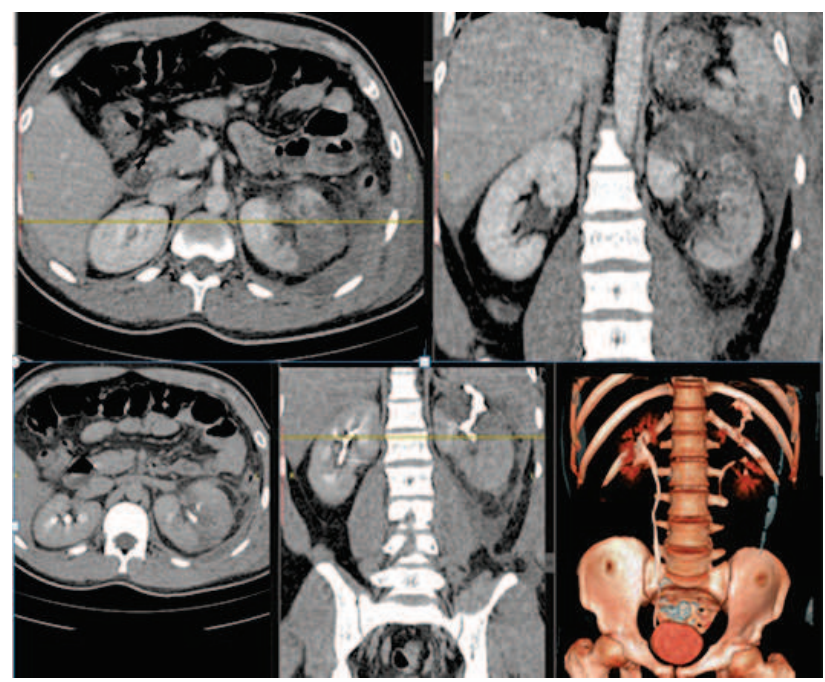




\section{Caso 5}

Paciente de 36 anos, sexo masculino, vítima de acidente motociclístico (Figura 5).

Achados Tomográficos: Laceração do polo inferior do rim direito. Lesão de hilo com extravasamento de urina em fase tardia. Hematoma perirrenal e retroperitoneal.

Classificação: Grau V.

Figura 5. Lesão renal grau V.

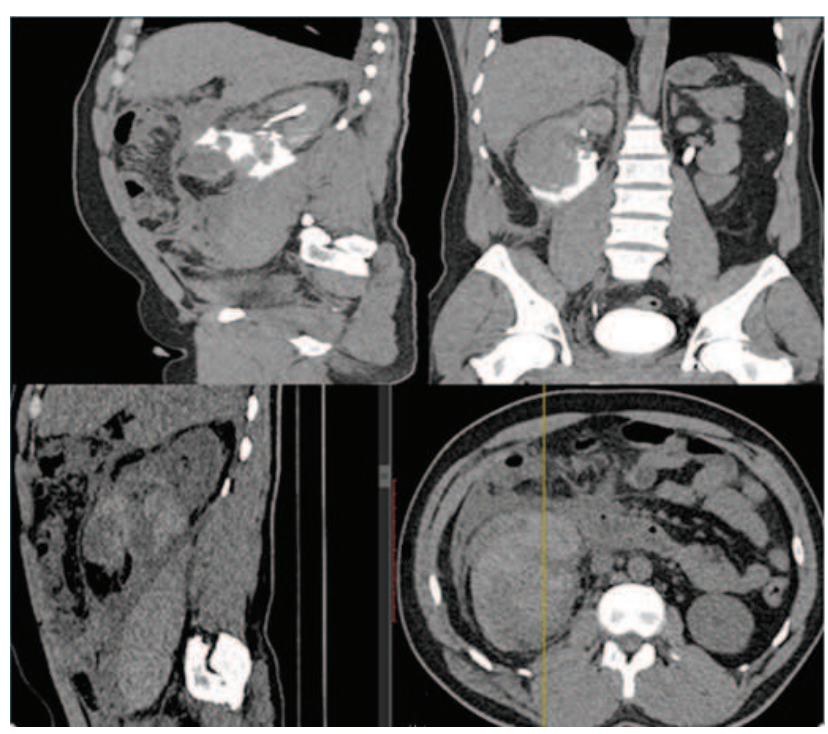

Embora o traumatismo renal ocorra com mais frequência em decorrência de trauma contuso (80-90\%), ${ }^{9}$ no Instituto Dr. José Frota, por se tratar de um hospital de referência em trauma e devido ao índice de violência urbana de nossa cidade, casos de traumatismo renal por ferimento penetrante, seja por arma

\section{REFERÊNCIAS}

1. Webb WR, Brant WE, Major N . Fundamentals of body CT. 4. ed. Philadelphia: Elsevier; 2015. 400 p.

2. Campbell DT, Somani BK. Renal trauma: case reports and overview. Case Rep Urol. 2012;2012:1-4.

3. Fonseca OC Neto, Vasconcelos R. Tratamento não cirúrgico do traumatismo renal contuso. J Bras Med. 2013;101(6):35-37.

4. Prando A, Moreira FA. Fundamentos de radiologia e diagnóstico por imagem. 2. ed. São Paulo: Elsevier; 2014. 872p.

5. Baghdanian AH, Baghdanian AA, Armetta A, Babayan RK, LeBedis CA, Soto JA, et al. Utility of MDCT findings in predicting patient management outcomes in renal trauma. Emerg Radiol. 2017;24(3):263-72.

6. Morey AF, Brandes S, Dugi DD 3rd, Armstrong JH, Breyer $\mathrm{BN}$, Broghammer JA, et al. Urotrauma: AUA guideline. J Urol. 2014;192(2):327-35. branca ou arma de fogo, não são infrequentes no nosso serviço, como nos dois casos relatados nesse estudo. Os outros três casos de trauma renal descritos foram decorrentes de acidentes de trânsito com motocicleta. O número de acidentes envolvendo moto é crescente e a elevada morbimortalidade deve-se a um conjunto de fatores como uma maior exposição corpórea. ${ }^{10}$

A grande maioria das lesões renais graus I, II e III são atualmente tratadas conservadoramente. Lesões grau IV e V são vistas em apenas 5\% dos traumas renais, quase sempre requerendo alguma forma de intervenção hemostática. A indicação absoluta para exploração cirúrgica é pequena: sangramento renal ativo, avulsão do pedículo renal, hematoma perirrenal pulsátil ou em expansão. Já indicações relativas de exploração cirúrgica são o extravasamento urinário, suspeita de área focal de infarto renal, diagnóstico tardio de lesão da artéria renal ou das artérias segmentares e dificuldade em classificar as lesões. ${ }^{9} \mathrm{O}$ extravasamento urinário isolado pode se resolver espontaneamente em mais de $70 \%$ dos casos, quando não resulta de lesão ureteral ou rotura pélvica.

Dos pacientes relatados neste estudo, todos foram tratados conservadoramente, exceto o paciente do caso 2 com ferimento por vários projéteis de arma de fogo, que foi abordado cirurgicamente, tendo sido submetido à laparotomia explorada, que revelou além das lesões renais já descritas anteriormente, lesão hepática no segmento II, lesão esplênica e várias lesões no jejuno. Foi então realizada a retirada de 20 $\mathrm{cm}$ de jejuno, enterorrafia e hemostasia com rafia da lesão renal encontrada. Diante do exposto acima, para um melhor manejo do paciente vítima de trauma renal, é necessária uma cuidadosa análise dos achados com descrição detalhada dos mesmos, afim de classificar corretamente a lesão renal, orientando o procedimento cirúrgico necessário, visando usar métodos terapêuticos menos invasivos possíveis. ${ }^{11}$

7. Lucchesi FR, Laguna CB, Monteiro CR, Prado CH, Elias J Jr. Diagnóstico por imagem no trauma abdominal. Medicina (Ribeirão Preto). 1999;32(4):401-18.

8. Mirvis SE, Shanmuganathan K. Abdominal computed tomography in blunt trauma. Semin Roentgenol . 1992;27(3):150-83.

9. Alonso RC, Nacenta SB, Martinez PD, Guerrero AS, Fuentes CG. Kidney in danger: $\mathrm{CT}$ findings of blunt and penetrating renal trauma. RadioGraphics. 2009;29(7):2033-53.

10. Batista SE, Baccani JG, Silva RA, Gualda KP, Vianna RJ Jr. Análise comparativa entre os mecanismos de trauma, as lesões e o perfil de gravidade das vítimas em Catanduva - SP. Rev Col Bras Cir. 2006;33(1):6-10.

11. Silva LF, Teixeira LC, Rezende JB Neto. Abordagem do trauma renal - artigo de revisão. Rev Col Bras Cir. 2009;36(6):519-24.

\section{Como citar:}

Moura FJ Filho, Oliveira M, Martins AM, Pontes PH, Barros MM. Trauma renal: ensaio pictórico dos principais achados tomográficos. Rev Med UFC. 2018 abr-jun;58(2):63-66. 